\title{
O SIGNIFICADO E A REPRESENTAÇÃO DA DOENÇA CRÔNICA: CONHECIMENTO DO PORTADOR DE HIPERTENSÃO ARTERIALACERCA DE SUA ENFERMIDADE*
}

\author{
Maria de Fátima Mantovani ${ }^{1}$, Elis Martins Ulbrich², Suzana Pinotti ${ }^{3}$ Letícia Morgana Giacomozzi ${ }^{4}$, Liliana Maria \\ Labronici $^{5}$, Leila Maria Mansano Sarquis ${ }^{5}$
}

\begin{abstract}
RESUMO: Trata-se de um estudo quanti-qualitativo realizado de novembro de 2005 a maio de 2008, em uma unidade de saúde da Prefeitura de Curitiba - PR. O objetivo do estudo foi descrever o conhecimento dos usuários inscritos e ativos do Programa de Hipertensão Arterial sobre a doença. Os participantes foram 69 usuários e os dados foram obtidos por meio de entrevista semi-estruturada. Do total de participantes, 45 eram do sexo feminino, 36 tinham idades entre 41 e 60 anos, e 37 com o ensino fundamental incompleto. Os usuários conheciam e definiam a doença, e sabiam das principais complicações, mas este fato não aumentou o conhecimento sobre a condição crônica da hipertensão. A existência da doença foi representada pela mudança da consistência do sangue, das artérias do coração e do estado emocional.

PALAVRAS-CHAVE: Hipertensão arterial; Doença crônica; Enfermagem.

\section{THE MEANING AND REPRESENTATION OF CHRONIC DISEASE: THE KNOWLEDGE OF HYPERTENSIVE INDIVIDUALS ON THEIR DISEASE}

ABSTRACT: It is a quanti-qualitative study held at a health center in Curitiba municipality/Brazil from November/2005 to May/2008. The study aimed to identify and describe the knowledge on the disease of active users enrolled in the hypertension program. Data were collected by means of a semi-structured interview with 69 users. Among the total participants, 45 were female users, 36 of them between 41 and 60 years of age, and 37 with non-concluded elementary educational level. The users knew, defined the disease and were aware of its main complications but this did not raise their knowledge of hypertension as a chronic condition. The existence of the disease to users was represented by changes in consistency of blood, heart arteries, and emotional state.

KEYWORDS: Hypertension; Chronic disease; Nursing.

\section{EL SIGNIFICADO Y LA REPRESENTACIÓN DE LA ENFERMEDAD CRÓNICA: CONOCIMIENTO DEL PORTADOR DE HIPERTENSIÓN ARTERIAL ACERCA DE SU ENFERMEDAD}

RESUMEN: El objetivo de este estudio fue identificar y describir el conocimiento de los usuarios inscritos y activos del Programa de Hipertensión Arterial de una unidad de salud de Curitiba, Paraná, acerca de la enfermedad. Se trata de un estudio cuantitativo-cualitativo, realizado en el período de noviembre de 2005 a mayo de 2008. Los datos fueron obtenidos por medio de entrevista semiestructurada, después de permisión del Comité de Ética, con 69 usuarios, siendo 45 del sexo femenino, 36 con edad entre 41 y 60 años y 37 con enseñanza fundamental incompleta. Los usuarios conocían y definían la enfermedad, y sabían de las principales complicaciones, pero esto no aumentaba el conocimiento acerca de la condición crónica de la hipertensión. La existencia de la enfermedad a los usuarios fue representada por los cambios en la consistencia de la sangre, las arterias del corazón y el estado emocional.

PALABRAS CLAVE: Hipertensión arterial; Enfermedad crónica; Enfermería.

\footnotetext{
*Este projeto foi financiado pela Fundação de Amparo à Pesquisa do Estado do Paraná-Fundação Araucária.

${ }^{1}$ Enfermeira. Doutora em Enfermagem. Docente do Curso Graduação e do Mestrado em Enfermagem da Universidade Federal do Paraná -UFPR. Coordenadora do Grupo de Pesquisa GEMSA.

${ }^{2}$ Acadêmica do Curso de Graduação em Enfermagem da UFPR. Membro do GEMSA. Bolsista PIBIC-CNPq.

${ }^{3}$ Enfermeira graduada na UFPR. Membro do GEMSA. Ex-bolsista IC voluntária.

${ }^{4}$ Enfermeira. Mestranda da UFPR. Membro do GEMSA.

${ }^{5}$ Enfermeiras. Doutoras em Enfermagem. Docentes do Curso de Graduação e do Mestrado em Enfermagem da UFPR. Membros do GEMSA.
}

Autor correspondente:

Maria de Fátima Mantovani

Rua Padre Camargo, 280 - 80060-240 - Curitiba-PR

E-mail: mantovan@ufpr.br. 


\section{INTRODUÇÃO}

As doenças crônicas representam a principal causa de mortalidade no mundo, principalmente as relativas às doenças cardiovasculares, que acarretam sérios problemas de incapacidade decorrentes de cardiopatias ou acidentes vasculares cerebrais. Dentre os 16,6 milhões de óbitos por doenças cardiovasculares que ocorrem a cada ano, 3,0 milhões se devem à hipertensão $\operatorname{arterial}^{(1)}$.

A hipertensão arterial acometia, em 2002, aproximadamente $22 \%$ da população adulta brasileira, e correspondia a $15,2 \%$ das intervenções realizadas no Sistema Único de Saúde (SUS) e de 20 a 30\% dos casos de emergência médica com sugestivas complicações ${ }^{(2)}$. Além disso, constitui o principal fator de risco para doenças cardiovasculares, as quais representam a primeira causa de mortalidade no Brasil, sendo desde a década de 60 , do século XX, mais comuns que as doenças infecciosas e parasitárias ${ }^{(3)}$.

A hipertensão pode ser controlada desde que o paciente se envolva e dê importância tanto para o tratamento medicamentoso como para o não medicamentoso, sendo também necessário o acompanhamento das co-morbidades como: o diabetes, a dislipidemia e a obesidade. Desta forma, há uma redução nas complicações em órgãos-alvo, que são conseqüências da hipertensão ao longo dos anos ${ }^{(4)}$.

A constituição desse processo impõe, portanto, a necessidade de mudar o estilo de vida e de tratar a doença sem interrupção, devido ao caráter crônico da doença que se caracteriza por uma história natural prolongada com uma multiplicidade de fatores de risco complexos. Em sua constituição interagem fatores etiológicos e biológicos conhecidos e desconhecidos, com curso clínico prolongado e permanente, com manifestações clínicas em períodos de remissão e exacerbação, podendo evoluir para graus variados de incapacidade e morte ${ }^{(5)}$.

Esses aspectos fazem com que a aceitação da convivência com o caráter crônico da enfermidade seja um grande desafio que está, muitas vezes, associado a sentimentos de tristeza, raiva e hostilidade cuja superação só se faz tornando possível uma adaptação à condição de saúde. Essa requer do indivíduo conhecimento relativo à doença, manifestações clínicas, sinais e sintomas, além de vontade de cooperar ativamente no tratamento. Porém, existem lacunas no conhecimento da doença e suas complicações e o "estar doente” para os sujeitos muitas vezes só é considerado após a instalação das complicações ou em situações que a hipertensão os impede de exercer as suas atividades cotidianas ${ }^{(6)}$.

Frente ao exposto, nesta pesquisa o objetivo foi descrever o conhecimento dos usuários de uma unidade de saúde sobre a hipertensão arterial.

\section{METODOLOGIA}

Estudo quanti-qualitativo desenvolvido em uma Unidade de Saúde da Prefeitura Municipal de CuritibaPMC, com início em novembro de 2005, após autorização dos Comitês de Ética do Setor de Ciências da Saúde da Universidade Federal do Paraná sob o n ${ }^{\circ}$ 008008509105 e da Prefeitura Municipal de Curitiba.

Participaram desta pesquisa 69 usuários, inscritos e ativos no Programa de Hipertensão Arterial, com idades entre 18 a 65 anos.

Os dados foram obtidos por meio de entrevista semi-estruturada, realizada no domicílio dos usuários. Foi utilizado para coleta de dados um formulário contendo dados de identificação (idade, sexo, pressão arterial, peso, altura, estado civil, escolaridade, renda familiar, hábitos e vícios, antecedentes familiares, tipo de tratamento já utilizado para hipertensão e em uso, conhecimento dos fatores de risco e das complicações, conhecimento das complicações já instaladas da doença e presença de co-morbidades) e questões abertas sobre o conhecimento da doença, como: definição de hipertensão arterial e de pressão alta, conhecimento sobre a cronicidade e das complicações da doença.

Os dados foram analisados em duas fases. Inicialmente, com a caracterização dos indivíduos pesquisados e, na seqüência, com a elaboração das categorias que foram estabelecidas mediante a transcrição e análise das entrevistas, realizadas segundo o método proposto por Bardin ${ }^{(7)}$, após a préanálise do material e a descrição analítica dos dados. As categorias estabelecidas foram: conhecimento da hipertensão arterial e pressão alta, da cronicidade, das complicações da doença e a representação do processo de adoecimento. Os trechos das entrevistas utilizados foram identificados com a letra B seguida do respectivo número.

\section{APRESENTAÇÃO E ANÁLISE DOS DADOS}

A Tabela 1 apresenta as características dos entrevistados, com destaque para as variáveis sexo, idade e escolaridade. 
Tabela 1 - Caracterização do perfil sócio-econômico dos hipertensos. Curitiba, 2005

\begin{tabular}{|c|c|c|}
\hline Variáveis & $\mathrm{N}^{\circ}$ & $\%$ \\
\hline \multicolumn{3}{|l|}{$\overline{\text { Sexo }}$} \\
\hline Masculino & 24 & $35 \%$ \\
\hline Feminino & 45 & $65 \%$ \\
\hline Total & 69 & $100 \%$ \\
\hline \multicolumn{3}{|l|}{ Faixa Etária } \\
\hline $31-40$ & 7 & $10 \%$ \\
\hline $41-50$ & 18 & $26 \%$ \\
\hline$>51$ & 44 & $64 \%$ \\
\hline Total & 69 & $100 \%$ \\
\hline \multicolumn{3}{|l|}{ Escolaridade } \\
\hline Analfabeto & 5 & $8 \%$ \\
\hline $1^{\mathrm{a}}$ a $4^{\mathrm{a}}$ série & 36 & $52 \%$ \\
\hline $5^{\mathrm{a}}$ a $8^{\mathrm{a}}$ série & 17 & $25 \%$ \\
\hline Médio Completo & 5 & $8 \%$ \\
\hline Médio Incompleto & 3 & $4 \%$ \\
\hline Superior & 3 & $4 \%$ \\
\hline Total & 69 & $100 \%$ \\
\hline \multicolumn{3}{|l|}{ Estado Civil } \\
\hline Solteiro & 6 & $9 \%$ \\
\hline Casado & 35 & $51 \%$ \\
\hline Amasiado & 4 & $6 \%$ \\
\hline Divorciado & 10 & $14 \%$ \\
\hline Viúvo & 14 & $20 \%$ \\
\hline Total & 69 & $100 \%$ \\
\hline \multicolumn{3}{|l|}{ Número de Filhos } \\
\hline nenhum filho & 5 & $8 \%$ \\
\hline 1 filho & 5 & $8 \%$ \\
\hline 2 filhos & 16 & $23 \%$ \\
\hline 3 filhos & 15 & $21 \%$ \\
\hline 4 filhos & 12 & $17 \%$ \\
\hline$>5$ filhos & 16 & $23 \%$ \\
\hline Total & 69 & $100 \%$ \\
\hline \multicolumn{3}{|l|}{ Renda Familiar } \\
\hline < 1 salário mínimo & 1 & $2 \%$ \\
\hline 1 a 3 salários mínimos & 42 & $61 \%$ \\
\hline 3 a 5 salários mínimos & 25 & $36 \%$ \\
\hline > 5 salários mínimos & 1 & $2 \%$ \\
\hline
\end{tabular}

Dos 69 hipertensos entrevistados, observamos que 45 são do sexo feminino e 24 do sexo masculino, corroborando com outros estudos ${ }^{(8-9)}$, nos quais também se observou que as mulheres aparecem em maior número. Em relação a esta questão ${ }^{(4)}$, não há indícios de que o sexo represente fator de risco, pois a prevalência global de hipertensão entre homens é 26,6\% e de $26,1 \%$ entre mulheres.

Pode-se dizer que os níveis tensionais tendem a se elevar em idades mais avançadas, fato este associado, geralmente, com o desenvolvimento da arteriosclerose, que leva à diminuição da complacência das artérias, ocasionando principalmente a hipertensão arterial sistólica isolada ${ }^{(4)}$. Entretanto, é importante ressaltar que $36 \%$ das pessoas que constituem a amostra encontram-se com idade inferior a 50 anos.

Ao analisar o nível de escolaridade verificouse que $52 \%$ dos indivíduos estudaram até a quarta série, fato relevante, pois este se configura como um fator que pode contribuir para dificultar ou facilitar a compreensão dos indivíduos acerca das orientações recebidas, sobretudo das medidas de tratamento correto $^{(9)}$.

A amostra caracteriza-se por ser de pessoas casadas (35), o que facilita a sua inserção no contexto da doença e do tratamento ${ }^{(4)}$. O suporte familiar (o apoio e a presença constante no tratamento, o acompanhamento às consultas, a lembrança do horário da medicação, o incentivo às alterações necessárias no estilo de vida e até mesmo a adesão conjunta ao tratamento) pode auxiliar a adesão do hipertenso ao tratamento e, consequentemente, evitar ou postergar as complicações da hipertensão arterial $^{(10)}$.

Os fatores de risco mais presentes nesse estudo foram: história familiar, sedentarismo e estresse. Quanto à história familiar, observa-se que os fatores genéticos interferem no desenvolvimento da hipertensão arterial, já que existe uma associação mais importante entre os níveis pressóricos entre irmãos biológicos, comparados aos de irmãos adotivos, demonstrando evidências de que os níveis de pressão arterial são, em parte, geneticamente determinados ${ }^{(11)}$.

O sedentarismo também é um fator de risco para a hipertensão arterial e foi evidenciado neste estudo, em que 31 dos entrevistados relataram nunca ter realizado nenhum tipo de exercício físico e 13 afirmaram também não desenvolver atividades de lazer. O exercício físico regular reduz a pressão arterial sistólica e diastólica em 3/2 Hg em normotensos, o que recomenda sua prática ${ }^{(4)}$. 
Quanto ao estresse, entrevistados admitem conviver com esses fatores diariamente, os quais são associados a problemas no trabalho, no lar e com os familiares. Em um estudo ${ }^{(12)}$, também foi observada uma alta incidência de respostas relacionadas a problemas em casa, além de contrariedade, raiva, ansiedade e irritação decorrentes da interação com outras pessoas. O estresse durante o trabalho, mencionado pelos entrevistados, é um dado relevante, já que 21 usuários exercem atividade remunerada.

Desta forma, "a preocupação social, econômica e familiar são fatores que elevam as cifras tencionais, merecendo atenção por parte dos profissionais da saúde no momento de se planejar qualquer atendimento que vá ao encontro das necessidades da população”.

\section{Categorias de análise}

\section{Conhecimento da hipertensão arterial (HA) e pressão alta}

As definições que os usuários possuem sobre hipertensão arterial e pressão alta estão relacionadas às alterações na consistência do sangue e às alterações na freqüência e força dos batimentos cardíacos:

O sangue tá agitado (B.47).

Dificuldade na circulação do sangue (B.52).

Quando está com o colesterol alto [...] (B.69).

Coração fica mais agitado, bate mais rápido (B.8).

Coração com dificuldade de bombear o sangue (B.52).

Os usuários definem a doença relacionando-a a alterações circulatórias e cardíacas, pois, quando não compreendem a real causa da doença que possuem, passam a defini-la com aspectos que conseguem assimilar e explicar. Aspectos semelhantes foram descritos em estudo ${ }^{(13)}$ no qual se evidenciou a associação da hipertensão arterial a problemas circulatórios e de desconforto físico.

Outra resposta dada para a definição de hipertensão e pressão alta foi relacionada aos sinais e sintomas da doença e ao nervosismo.

Fico tonta, zonza, sinto dor de cabeça (B.13).

Quando sobe a pressão dá dor na nuca (B.49).
Sinto dor na nuca, dor no corpo [...] (B.8).

É eu ficar nervosa, a pressão vai lá em cima [...] (B.21).

Sobe por causa dos nervos (B.39).

A definição da hipertensão arterial pelos seus sinais e sintomas também foi evidenciada em outro estudo $^{(13)}$, no qual os entrevistados associavam a doença aos desconfortos causados pela mesma, como: cefaléia, tontura, palpitação, insônia e outras.

Todavia, a hipertensão arterial raramente manifesta algum sintoma ou desconforto físico, sendo conhecida por isso como "assassina silenciosa”. Isso constitui uma das razões do portador não se comprometer com as condutas necessárias ao seu controle, pois as pessoas só se percebem doentes quando qualquer alteração na qualidade de vida as impede de realizar suas atividades rotineiras ${ }^{(13)}$.

Quando a doença manifesta algum sintoma, este pode estar relacionado à instalação de uma crise hipertensiva, o que corrobora com o identificado no estudo $^{(14)}$ no qual os usuários referiram sintomas como turvamento da visão e fraqueza. Este fato é definido como hipertensão arterial e pressão alta pelos usuários porque é o momento em que sentem que algo não está certo, que está causando incômodo. Verificouse, ainda, que são em situações estressantes que os indivíduos percebem o aumento dos níveis tensionais, fato também encontrado em outro estudo ${ }^{(12)}$, o qual evidenciou que é freqüente os usuários rotularem a doença hipertensiva de "emocional” e "nervosa", mostrando um reducionismo na atribuição das causas da hipertensão, o que demonstra um desconhecimento da natureza multifatorial da doença.

A relação da doença com as complicações e o risco iminente de morte também foi encontrada na fala dos sujeitos, conforme se observa abaixo:

Cegueira, enfarte, derrame e morte (B.66).

Pode levar a um ataque cardíaco e derrame (B.62).

Perigoso [...] pode dar derrame e enfarte (B.24).

\section{Conhecimento sobre a cronicidade da doença}

Quando indagados se a hipertensão arterial tem cura, um grupo de usuários referiu que a doença tem apenas controle, pois nunca constataram alguém parar de tomar os remédios. 
Não, mesmo controlada não cura (B.65).

Não, porque nunca vi ninguém parar de tomar o remédio (B.61).

Não, só controle, porque se relaxar já volta (B.8).

A noção de que a doença às vezes tem cura com o uso de medicamentos pode ser vista na fala de B.8; este usuário demonstra dúvidas sobre a possível cura da doença, pois acredita que a falta de medicamentos/tratamento faz com que ela retorne. Verifica-se que a noção de cronicidade da doença entre estes usuários não está clara, sendo que alguns usuários afirmam que a doença possui cura. Segundo estudo(15), a maior dificuldade para a equipe de saúde é conscientizar a população para aceitar a condição crônica da doença.

Tratando-se da cura da hipertensão alguns usuários referiram:

Só Deus que pode curar ela; se Deus der a cura, a gente sara (B.4).

Sim, se cuidar cura (B.1).

Sim, cura do Senhor (B.19).

Sim, porque hoje estou bem (B.32).

Não, só se for numa curandeira (B.12).

Acho que sim. Fazendo dieta e tomando o remédio (B.6).

Quando se acredita que a hipertensão tem cura, ela é atribuída à divindade, à magia ou ao tratamento bem feito. Percebe-se a necessidade de esclarecimentos quanto à doença, pois quanto maior 0 grau de conhecimento, maior a possibilidade de aceitação da condição de saúde, aumentando o comprometimento ao tratamento.

Alguns usuários afirmaram que a doença tem cura se o tratamento for realizado corretamente.

Sim, se cuidar cura (B.1).

Acho que sim [...] se fizer dieta e tomar os remédios (B.6).

Sim, porque hoje estou bem (B.32).

Cura se fizer o tratamento direito (B.41).
Percebe-se, nos trechos anteriores, que os usuários deste grupo ainda não têm consciência do caráter crônico da hipertensão arterial e da necessidade da realização do tratamento anti-hipertensivo para o resto de suas vidas. Esses sujeitos são mais suscetíveis às complicações da doença, já que acreditam que na ausência de sinais e sintomas não necessitam continuar a terapêutica. Um dos maiores obstáculos a ser enfrentado pelos portadores de hipertensão arterial é aceitar a convivência com a doença, cuja superação é dada através da conscientização de sua nova condição de vida e da necessidade de controle da saúde ${ }^{(9)}$.

\section{Conhecimento das complicações da doença}

Quanto ao conhecimento das complicações da hipertensão arterial, as mais citadas foram o infarto e o derrame, seguidas da cegueira e paralisação dos rins. Outra resposta encontrada para esta questão foi o medo da morte, já que muitos responderam que a hipertensão arterial pode matar, contrariando os resultados de um estudo $^{(16)}$, no qual esta associação com a morte não ocorreu, dificultando a mudança nos hábitos de vida.

Entupimento das veias, enfarte e derrame (B.9.)

Derrame cerebral, paralisação dos rins, problema cardíaco (B.14).

Pode dar enfarte e derrame (B.19).

Derrame [...], ficar inválido (B.55).

Problemas no rim [...] visão dói (B.8).

Pode morrer (B.54).

As complicações mais conhecidas pelos pacientes sobre a doença hipertensiva foram derrame e infarto, dados encontrados em outro estudo ${ }^{(12)}$, no qual os pacientes hipertensos referiram medo quando a pressão estava alta, pois este fato poderia acarretar um derrame e com isto eles passariam a depender dos outros. Apenas alguns usuários se referiram às complicações renais e visuais, o que parece indicar que os sujeitos possuem um conhecimento parcial sobre as conseqüências da hipertensão arterial. Mas, este reconhecimento é um aspecto que pode favorecer a adoção de comportamentos positivos em relação aos cuidados com a doença. Embora a doença seja caracterizada como assassina silenciosa, esse "silêncio", muitas vezes, é interpretado como ausência

Cogitare Enferm 2008 Jul/Set; 13(3):336-42 
de patologia, levando as pessoas a se perceber como hipertensas somente no surgimento de sintomas ou complicação desta ${ }^{(13)}$.

\section{Representação do processo de adoecimento}

A representação que estes usuários fazem do processo de adoecimento e da hipertensão é de que para a doença existir seria necessário que o corpo se alterasse, quer na consistência do sangue, das artérias, do estado emocional ou do coração:

Quando o sangue tá grosso, as veias fecham aí não tratando a pressão, fica com diabetes (B.14).

É eu ficar nervosa a pressão vai lá em cima. Não consigo controlar com a medicação (B.21).

Quando sobe a temperatura ou desce a temperatura do corpo (B.16).

Força do coração; coração bate mais forte (B.33). Gordura na veia (B.46).

A representação da hipertensão ${ }^{(16)}$ surge como um mal que pode ser controlado com medicamento e que, se controlada, "não mata”, sendo fator preocupante somente quando há um sintoma. Também é notada na fala de um usuário, em outro estudo, no qual há o desconhecimento do significado da pressão alta e há uma representação social na qual ela aparece associada a uma doença crônica que avança no tempo, e que os danos aparecem "numa temporalidade distante".

\section{CONSIDERAÇÕES FINAIS}

Os resultados do estudo demonstram um déficit de conhecimento dos usuários acerca da hipertensão arterial, já que possuem crenças em relação à doença associadas a um conceito parcial sobre alguns de seus aspectos. A doença foi definida com aspectos do imaginário, com associações a sinais, sintomas e ao nervosismo.

Contudo, evidenciou-se a ciência das complicações da hipertensão, principalmente o infarto agudo do miocárdio e o acidente vascular cerebral. Todavia, este fato pode não implicar em maior adesão à terapêutica, pois os entrevistados não reconheciam a cronicidade da doença e a necessidade de tratamento contínuo.
Portanto, é necessário buscar estratégias que permitam maior conhecimento da doença e de suas complicações, como a educação em saúde. O processo educativo deve abranger aspectos que permitam: conhecer as atitudes, percepções e práticas do portador de hipertensão; incentivar a participação ativa destes no tratamento; levar em consideração suas dificuldades e necessidades; estabelecer adequada comunicação e interação entre os envolvidos no processo educativo; trabalhar os aspectos cognitivos e psicossociais dos usuários e buscar o envolvimento da família no tratamento do portador de hipertensão arterial $^{(12)}$.

Com estas iniciativas acreditamos facilitar a aceitação da doença, melhorar e reduzir as complicações decorrentes da hipertensão mal controlada. Todavia, para atingir esses objetivos é necessário uma abordagem multiprofissional ao hipertenso, que ocorra de forma contínua, contemplando todos os aspectos do tratamento anti-hipertensivo, a fim de manter a qualidade de vida dos sujeitos.

\section{REFERÊNCIAS}

1. Organização Pan-Americana da Saúde(OPAS). Cuidados inovadores para condições crônicas: componentes estruturais da ação. Brasília; 2003.

2. Almeida Filho N, Rouquayrol MZ. Introdução à epidemiologia. Rio de Janeiro: Medsi, 2002.

3. Brasil. Ministério da Saúde. Secretaria de Políticas de Saúde. Plano de reorganização da atenção à hipertensão arterial e ao diabetes mellitus. Brasília; 2002.

4. Sociedade Brasileira de Hipertensão. V Diretrizes Brasileiras de Hipertensão Arterial. 2006. Disponível em: http//www.sbh.org.br//. Acesso em 31 Ago 2008.

5. Lessa I. O adulto brasileiro e as doenças da modernidade: epidemiologia das doenças crônicas não transmissíveis. São Paulo: Hucitec; 1998.

6. Ribeiro DS, Mantovani MF. Caminhando para a cronicidade. Traduzido do original: Walking to chronicity: representations of illness in adults with angina pectoris. Cogitare Enferm. 2001;6(1):97-104.

7. Bardin L. Análise de conteúdo. Lisboa: Edições 70; 1977.

8. Pierin AMG, Mion JRD, Fukushima JT, Pinto AR, Kaminaga MM. O perfil de um grupo de pessoas hipertensas de acordo com conhecimento e gravidade 
da doença. Rev Esc Enferm USP. 2001;35(1):11-8.

9. Fortes AN, Lopes VO. Análise dos fatores que interferem no controle da pressão arterial de pessoas acompanhadas numa unidade básica de atenção à saúde da família. Texto Contexto Enferm. 2004;13(1):26-34.

10. Lima FET; Lopes MVO; Araújo TL. A família como suporte para o idoso no controle da pressão arterial. Fam Saúde Desenv. 2001;3(1):63-9.

11. Pereira AC, Krieger JE. Dos fatores de risco clássicos ao perfil de risco individualizado: quais são os caminhos? Rev Soc Bras Hipert [periódico na internet]. 2005 [acesso em 2008 Abr 15]8(4):131-7. Disponível em: www.sbh.org.br.

12. Péres DS, Magna JM, Viana LA. Portador de hipertensão arterial: atitudes, crenças, percepções, pensamentos e práticas. Rev Saúde Públ. 2003;37(5): 642-53.

13. Santos ZMSA, Frota MA, Cruz DM, Holanda SD. Adesão do cliente hipertenso ao tratamento: uma analise com abordagem interdisciplinar. Texto Contexto Enferm. 2005;14(3):332-40.

14. Mantovani MF, Mottin JV, Rodrigues J. Nurse home visity with educational activities in the arterial pressure treatment. Online Brazilian Journal of Nursing [periódico na internet]. 2007 [acesso em 2008 Abr 15]6(2). Disponível em www.uff.br/objnursing.

15. Vilella JC. A percepção das complicações da hipertensão arterial e a condição crônica de saúde [monografia]. Curitiba(PR): Universidade Federal do Paraná; 2006.

16. Rey FLG. As representações sociais como produção subjetiva: seu impacto na hipertensão e no câncer. Psicol Teoria Prat. 2006; 8(2):69-85. 